\title{
Perforated jejunal diverticulitis: a rare but important differential in the acute abdomen
}

\author{
Natasha Leigh ${ }^{1^{*}}$ D, Brianne J. Sullivan ${ }^{1}$, Roi Anteby ${ }^{2}$ and Susan Talbert ${ }^{1}$
}

\begin{abstract}
Background: Diverticulosis of the small bowel is rare and, in most cases, discovered incidentally. However, diverticulitis and other complications are important to consider in the differential of an acute abdomen, especially in the elderly population.

Case presentation: The patient was a 59-year-old female who presented with acute lower abdominal pain progressing to peritonitis. Computed tomography scan showed a large inflamed and perforated diverticulum on the mesenteric side of the jejunum. Exploratory laparotomy revealed a dilated proximal jejunum with a 5-cm inflamed and perforated mesenteric diverticulum. A small bowel resection with primary anastomosis was performed.

Conclusions: Jejunal diverticulitis remains a diagnostic challenge. Although uncommon, owing to its high mortality rate, it is an important clinical entity to consider and requires timely management.
\end{abstract}

Keywords: Jejunal diverticulitis, Abdominal pain, Acute abdomen, Perforation, Diverticulitis

\section{Background}

Jejunal diverticulosis is a relatively rare condition with a reported annual incidence of $0.3-2.3 \%$ [1]. The majority of cases are asymptomatic and found incidentally either on computed tomography $(\mathrm{CT})$ scan or at the time of operation for an unassociated condition. In a percentage of patients, akin to colonic diverticulosis, they can become acutely inflamed (diverticulitis) or have a more complicated presentation including perforation, intestinal bleeding, or obstruction [2]. Owing to the rarity of this condition and varied presentation, clinical diagnosis alone remains challenging and adjunctive imaging techniques are commonly required in order to form a prompt diagnosis.

\section{Case presentation}

A 59-year-old female presented to the emergency department with $48 \mathrm{~h}$ of sudden onset abdominal pain. The pain began across the lower abdomen and subsequently became diffuse involving all four quadrants. Associated symptoms included nausea without vomitus and fever. Her past medical history was significant for hypertension, type II diabetes mellitus, depression, and one episode of colonic diverticulitis managed with antibiotics alone. Vital signs were abnormal with a fever of $102^{\circ} \mathrm{F}$ and tachycardia of $121 \mathrm{bpm}$, but normotensive $129 / 75 \mathrm{mmHg}$. On physical examination, the patient was tender to palpation throughout the lower and mid abdomen without rebound or guarding and had some fullness over the umbilical region.

\section{Diagnostic studies}

Laboratory results were notable for an elevated white blood cell count (15.3) with $81 \%$ neutrophilia. All other labs were within normal limits including blood urea
* Correspondence: tashleigh88@gmail.com Luke's Roosevelt Hospital, 425 West 59th Street, Suite 7B, New York, NY 10019, USA

Full list of author information is available at the end of the article (c) The Author(s). 2020 Open Access This article is licensed under a Creative Commons Attribution 4.0 International License, which permits use, sharing, adaptation, distribution and reproduction in any medium or format, as long as you give appropriate credit to the original author(s) and the source, provide a link to the Creative Commons licence, and indicate if changes were made. The images or other third party material in this article are included in the article's Creative Commons licence, unless indicated otherwise in a credit line to the material. If material is not included in the article's Creative Commons licence and your intended use is not permitted by statutory regulation or exceeds the permitted use, you will need to obtain permission directly from the copyright holder. To view a copy of this licence, visit http://creativecommons.org/licenses/by/4.0/. 
nitrogen (BUN) (13), creatinine (0.92), and lactic acid (1.27).

A CT scan of the abdomen with oral and intravenous (IV) contrast revealed a jejunal loop with a large diverticulum on the mesenteric side with associated diverticulitis and a $5.3 \times 3.6 \times 4.8 \mathrm{~cm}$ contained perforation. There was extensive edema of the entire jejunal loop and hazy infiltration of the adjacent mesentery (Fig. 1a, b). No arrowhead sign (an arrowhead-shaped collection of extraluminal air packed between the perforated diverticulum and inflamed mesentery) was seen in this case. There was also no lymphadenopathy or ascites seen. There were also multiple duodenal, jejunal, ileal, and colonic diverticula without evidence of diverticulitis.

The patient was admitted with the diagnosis of jejunal diverticulitis with perforation but without clinical peritonitis. She was taken emergently to the operating room and underwent an exploratory laparotomy via midline incision. On entering the abdomen, a small amount of serous fluid was encountered without frank purulence. The involved jejunal loop was found to have serosal purulent exudates as well as interloop adhesions. The proximal jejunum was dilated and the distal jejunum collapsed. Twenty-seven centimeters of the proximal jejunum was resected to healthy non-inflamed edges, and a stapled side-to-side functional end-to-end anastomosis was performed. The small bowel was run from the ligament of Treitz to the ileocecal junction, and 4 other large non-inflamed diverticula ranging from 0.5 to $3 \mathrm{~cm}$ in diameter were seen along the mesenteric border. These were widely spaced and therefore not excised, as this would have required multiple further small bowel resections and anastomoses with associated increased morbidity. The abdomen was lavaged and closed.

\section{Pathology}

Gross examination of the specimen revealed a $27-\mathrm{cm}$ length of the jejunum with focal areas of fibrinous material. Within the specimen were three diverticula originating from the mesenteric side of the jejunal serosa. On microscopic examination, one diverticulum demonstrated transmural inflammation with acute inflammatory exudate and perforation. The other two appeared non-inflammatory. The surrounding omentum was thickened with fibrinous exudates and focal areas of hemorrhage (Fig. 2a, b).

\section{Postoperative course}

Postoperatively, the patient's course was uncomplicated. She had return of bowel function, tolerated a regular diet, and was discharged on postoperative day 6 .

\section{Discussion}

Diverticular disease is a relatively common disorder and may be multifocal throughout the intestinal tract. Most frequently, it affects the colon; however, other sites include the jejunum, ileum, and duodenum. Jejuno-ileal diverticulosis was first described in 1794 by Somerling. It is relatively rare, comprising only $18 \%$ of all small bowel diverticula, which is likely an underestimate given they are often incidentally found on imaging [3-5]. Their etiology is unclear; however, some studies have postulated that abnormal neuromotor innervation causing intestinal dyskinesia may be a factor [6]. This causes abnormally elevated intraluminal pressure resulting in the formation of false (pulsion) diverticula at points of weakness along the intestine where blood vessels penetrate the wall, similar to colonic diverticulosis. Unlike their true counterparts (to include Meckel's diverticula), they involve only the mucosal and submucosal layers of
A

B

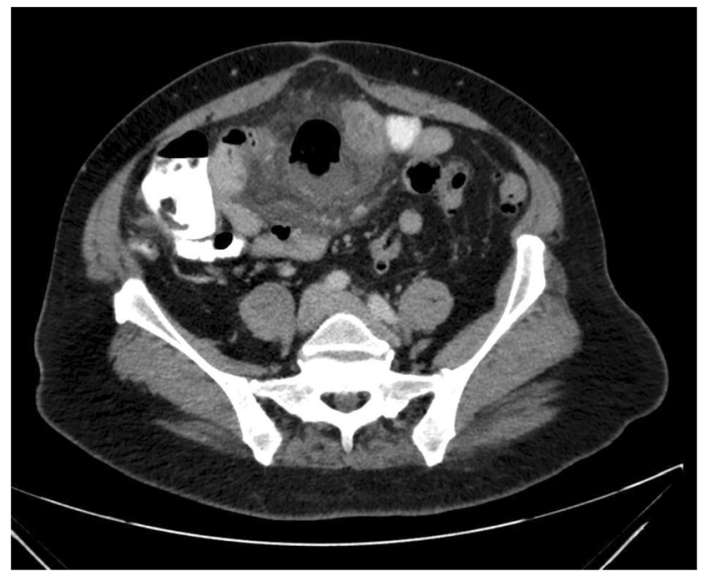

Fig. 1 CT of the abdomen with oral and IV contrast. a Axial image of the mid-jejunal diverticulum with perforation. b Coronal image demonstrating associated edema of the jejunal loop and adjacent mesentery 

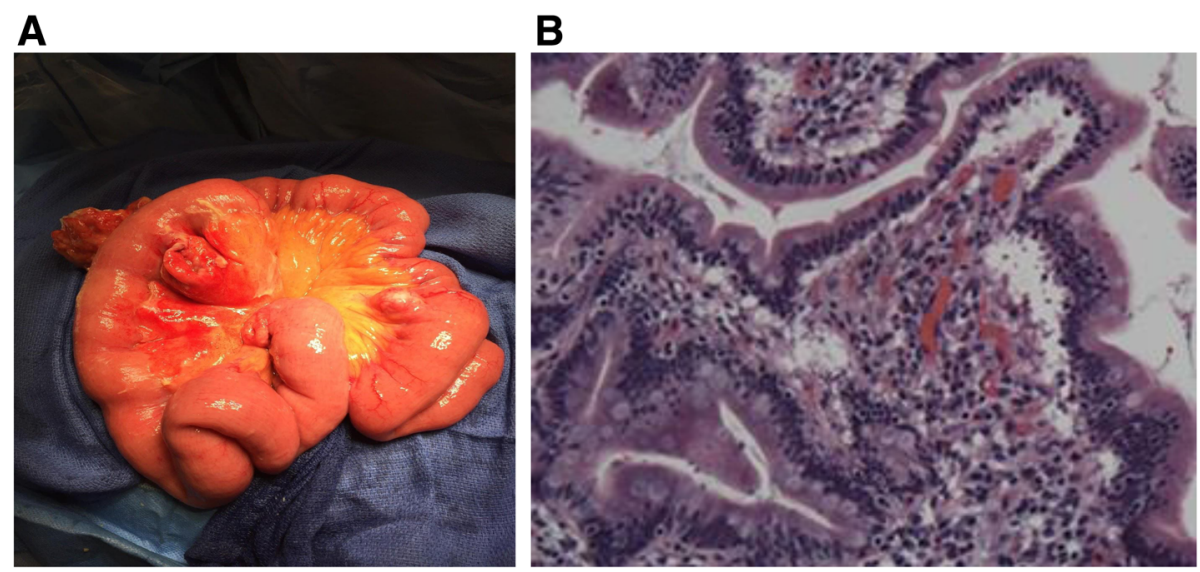

Fig. 2 Pathology. a Gross specimen of the resected jejunal segment containing the perforated diverticulum. b Histologic examination with transmural jejunal inflammation, serosal inflammatory exudate, and perforation

the bowel wall and are typically located on the mesenteric border [5, 7]. There appears to be an association with age older than 60 years, male gender, colonic diverticulosis, and systemic connective tissue diseases [2, 6]. Familial tendencies have also been noted.

Table 1 demonstrates cases of jejunal diverticulosis with diagnosis and management reported in the literature over the last 10 years. Comparing this case to others reported in literature, our patient was younger in age (mean age $=74$ ). She presented with an acute abdomen, whereas most small bowel diverticula are asymptomatic. The diverticula were found on the mesenteric side of the bowel, the most common location for jejunal diverticulosis. In cases published in the last decade, only one report stated finding anti-mesenteric small bowel diverticula [40]. Our patient was treated with an open surgical resection, as reported in the majority of the cases. Of note, there were reports of conservative treatment and laparoscopic resection $[5,7,9,12,14,17,19$, $25,28,31$, but not in patients with perforated diverticulitis presenting with an acute abdomen.

In the majority of cases, jejunal diverticulosis is diagnosed incidentally either on imaging or intraoperatively. However, around $10-30 \%$ of patients present with disease complications including diverticulitis, perforation, bleeding, or small bowel obstruction [46]. Isolated perforation is extremely rare, and to date, only a few cases have been reported in the English literature. Consequently, most centers have little experience with managing these cases. It is believed that in the same manner that asymptomatic colonic diverticulosis is managed without intervention, incidental jejunal diverticulosis is not of clinical significance. However, the diagnosis and management of its complications remains a challenge.
Patients typically present with non-specific symptomatology including acute abdominal pain in varied locations, fever, intestinal bleeding, and obstructive symptoms meaning that there is reliance on adjunctive tools such as CT scans or balloon enteroscopy and often a delay in making the correct diagnosis [47]. Many have adopted management strategies with a similar approach to colonic disease. Non-operative treatment with bowel rest and antibiotics has been successful for cases of uncomplicated diverticulitis [5]. However, in patients who present with acute peritonitis, hemodynamic instability, or evidence of free perforation, an aggressive operative approach is most appropriate. On review of complicated diverticulitis cases to date, most authors have had successful outcomes with resection of the involved small bowel segment and primary anastomosis [4, 46, 48]. This is especially important when considering the high mortality (around 20-30\%) associated with this disease process, mostly attributable to a delay in diagnosis. Our patient was managed in this manner and successfully discharged without the development of complications.

\section{Conclusion}

Complicated jejunal diverticulitis can be both a diagnostic and therapeutic challenge with a high mortality rate. We recommend that jejunal diverticulitis be considered a differential diagnosis in the acute abdomen and a CT scan will allow for the timeliest diagnosis. In cases of free perforation, operative resection of the affected segment with primary anastomosis appears to be a successful management strategy. 
Table 1 Characteristics and treatment of jejunal diverticulosis in cases published between 2010 and 2020

\begin{tabular}{|c|c|c|c|c|c|c|c|c|c|c|c|}
\hline Paper & Year & Age & Gender & $\begin{array}{l}\text { Preoperative } \\
\text { diagnosis }\end{array}$ & $\begin{array}{l}\text { Distance from } \\
\text { Trietz's lig. }(\mathrm{cm})\end{array}$ & Location & $\begin{array}{l}\text { No. of } \\
\text { diverticula }\end{array}$ & $\begin{array}{l}\text { No. of } \\
\text { perforations }\end{array}$ & Type & Diverticulitis & Treatment \\
\hline Prough H [8] & 2019 & 65 & $M$ & Diverticulitis & NA & Mesentery & 1 & 0 & NA & Yes & $\begin{array}{l}\text { Open surgery with } \\
\text { resection }\end{array}$ \\
\hline Gurala D [9] & 2019 & 70 & F & $\begin{array}{l}\text { Small intestine } \\
\text { diverticulitis }\end{array}$ & NA & NA & 2 & 1 & NA & Yes & $\begin{array}{l}\text { Failed conservative, } \\
\text { laparoscopic surgery } \\
\text { with resection }\end{array}$ \\
\hline $\begin{array}{l}\text { Mazahreh TS } \\
\text { [10] }\end{array}$ & 2019 & 68 & M & $\begin{array}{l}\text { Gastrointestinal } \\
\text { bleed }\end{array}$ & 10 & NA & Multiple & 0 & $\begin{array}{l}\text { True and } \\
\text { false }\end{array}$ & No & $\begin{array}{l}\text { Open surgery with } \\
\text { resection }\end{array}$ \\
\hline Fleres F [11] & 2018 & 88 & $\mathrm{~F}$ & Unknown & NA & NA & 3 & 1 & NA & Yes & $\begin{array}{l}\text { Open surgery with } \\
\text { resection }\end{array}$ \\
\hline Fleres F [11] & 2018 & 86 & F & Volvulus & 150 & Mesentery & Multiple & 0 & NA & Yes & $\begin{array}{l}\text { Open surgery with } \\
\text { resection }\end{array}$ \\
\hline $\begin{array}{l}\text { Abdelbaki A } \\
\text { [12] }\end{array}$ & 2018 & 65 & $\mathrm{~F}$ & $\begin{array}{l}\text { Small intestine } \\
\text { diverticulitis }\end{array}$ & 45 & NA & Multiple & 1 & NA & Yes & $\begin{array}{l}\text { Open surgery with } \\
\text { resection }\end{array}$ \\
\hline $\begin{array}{l}\text { Abdelbaki A } \\
{[12]}\end{array}$ & 2018 & 74 & $\mathrm{~F}$ & $\begin{array}{l}\text { Small intestine } \\
\text { diverticulitis }\end{array}$ & NA & NA & NA & 0 & NA & Yes & Conservative \\
\hline $\begin{array}{l}\text { Abdelbaki A } \\
{[12]}\end{array}$ & 2018 & 87 & M & $\begin{array}{l}\text { Small intestine } \\
\text { diverticulitis }\end{array}$ & NA & NA & NA & 0 & NA & Yes & Conservative \\
\hline Syllaios A [13] & 2018 & 75 & M & $\begin{array}{l}\text { Small intestine } \\
\text { diverticulitis }\end{array}$ & NA & NA & 6 & 0 & False & Yes & $\begin{array}{l}\text { Open surgery with } \\
\text { resection }\end{array}$ \\
\hline $\begin{array}{l}\text { Kagolanu DC } \\
\text { [5] }\end{array}$ & 2018 & 91 & M & $\begin{array}{l}\text { Small intestine } \\
\text { diverticulitis }\end{array}$ & NA & NA & Multiple & 0 & NA & Yes & Conservative \\
\hline Ejaz S [14] & 2017 & 76 & M & $\begin{array}{l}\text { Small intestine } \\
\text { diverticulitis }\end{array}$ & NA & NA & Multiple & NA & NA & Yes & Conservative \\
\hline Ejaz S [14] & 2017 & 78 & $\mathrm{~F}$ & $\begin{array}{l}\text { Small intestine } \\
\text { diverticulitis }\end{array}$ & NA & NA & Multiple & 0 & NA & Yes & Conservative \\
\hline Ejaz S [14] & 2017 & 87 & M & $\begin{array}{l}\text { Small intestine } \\
\text { diverticulitis }\end{array}$ & NA & Mesentery & Multiple & NA & NA & Yes & Conservative \\
\hline Grubbs J [15] & 2017 & 90 & M & $\begin{array}{l}\text { Sigmoid } \\
\text { diverticulitis }\end{array}$ & NA & NA & Multiple & 1 & NA & Yes & $\begin{array}{l}\text { Open surgery with } \\
\text { resection }\end{array}$ \\
\hline Kumar D [16] & 2017 & 60 & $\mathrm{~F}$ & $\begin{array}{l}\text { Small intestine } \\
\text { diverticulitis }\end{array}$ & NA & Mesentery & Multiple & 0 & NA & Yes & $\begin{array}{l}\text { Open surgery with } \\
\text { resection }\end{array}$ \\
\hline Kumar D [16] & 2017 & 68 & M & $\begin{array}{l}\text { Small intestine } \\
\text { diverticulitis }\end{array}$ & NA & Mesentery & Multiple & 1 & NA & Yes & $\begin{array}{l}\text { Open surgery with } \\
\text { resection }\end{array}$ \\
\hline Cui J [17] & 2017 & 65 & $\mathrm{~F}$ & $\begin{array}{l}\text { Small intestine } \\
\text { diverticulitis }\end{array}$ & NA & Mesentery & Multiple & 0 & NA & Yes & $\begin{array}{l}\text { Failed conservative, } \\
\text { laparoscopic surgery } \\
\text { with resection }\end{array}$ \\
\hline Malghan L [18] & 2017 & 91 & $\mathrm{~F}$ & $\begin{array}{l}\text { Small intestine } \\
\text { diverticulitis }\end{array}$ & NA & NA & Multiple & 0 & NA & Yes & $\begin{array}{l}\text { Open surgery with } \\
\text { resection }\end{array}$ \\
\hline Karas L [19] & 2017 & 82 & $\mathrm{~F}$ & Intestinal mass & NA & NA & Multiple & 0 & NA & Yes & $\begin{array}{l}\text { Open surgery with } \\
\text { resection }\end{array}$ \\
\hline Karas L [19] & 2017 & 80 & $\mathrm{~F}$ & $\begin{array}{l}\text { Small intestine } \\
\text { diverticulosis }\end{array}$ & NA & NA & Multiple & 0 & NA & Yes & $\begin{array}{l}\text { Laparoscopic surgery } \\
\text { with resection }\end{array}$ \\
\hline Mohi RS [20] & 2016 & 62 & M & Volvulus & NA & Mesentery & Multiple & 0 & NA & Yes & $\begin{array}{l}\text { Open surgery with } \\
\text { resection }\end{array}$ \\
\hline Aydin E [21] & 2016 & 69 & M & $\begin{array}{l}\text { Small intestine } \\
\text { diverticulitis }\end{array}$ & 20 & Mesentery & Multiple & 0 & NA & Yes & $\begin{array}{l}\text { Open surgery with } \\
\text { resection }\end{array}$ \\
\hline Tenreiro N [22] & 2016 & 81 & M & Diverticulitis & NA & NA & Multiple & 1 & NA & Yes & $\begin{array}{l}\text { Failed conservative, } \\
\text { open surgery } \\
\text { with resection }\end{array}$ \\
\hline Ghrissi R [23] & 2016 & 72 & M & $\begin{array}{l}\text { Small bowel } \\
\text { obstruction }\end{array}$ & NA & NA & Multiple & 0 & NA & No & $\begin{array}{l}\text { Open surgery with } \\
\text { resection }\end{array}$ \\
\hline Harbi H [24] & 2016 & 31 & M & Unknown & NA & NA & Multiple & 1 & False & Yes & $\begin{array}{l}\text { Open surgery with } \\
\text { resection }\end{array}$ \\
\hline $\begin{array}{l}\text { De Minicis S } \\
{[25]}\end{array}$ & 2015 & 60 & M & Jejunal diverticula & NA & NA & Multiple & 0 & NA & Yes & Conservative \\
\hline Natarajan K [26] & 2015 & 56 & M & $\begin{array}{l}\text { Small intestine } \\
\text { diverticulitis }\end{array}$ & 8 & Mesentery & Multiple & 3 & False & Yes & $\begin{array}{l}\text { Open surgery with } \\
\text { resection }\end{array}$ \\
\hline Kassir R [27] & 2015 & 79 & M & $\begin{array}{l}\text { Small intestine } \\
\text { diverticulitis }\end{array}$ & NA & Mesentery & Multiple & 1 & NA & Yes & $\begin{array}{l}\text { Open surgery with } \\
\text { resection }\end{array}$ \\
\hline
\end{tabular}


Table 1 Characteristics and treatment of jejunal diverticulosis in cases published between 2010 and 2020 (Continued)

\begin{tabular}{|c|c|c|c|c|c|c|c|c|c|c|c|}
\hline Paper & Year & Age & Gender & $\begin{array}{l}\text { Preoperative } \\
\text { diagnosis }\end{array}$ & $\begin{array}{l}\text { Distance from } \\
\text { Trietz's lig. }(\mathrm{cm})\end{array}$ & Location & $\begin{array}{l}\text { No. of } \\
\text { diverticula }\end{array}$ & $\begin{array}{l}\text { No. of } \\
\text { perforations }\end{array}$ & Type & Diverticulitis & Treatment \\
\hline Fidan N [7] & 2015 & 67 & M & $\begin{array}{l}\text { Small intestine } \\
\text { diverticulitis }\end{array}$ & NA & NA & Multiple & 0 & NA & Yes & Conservative \\
\hline Levack MM [28] & 2014 & 77 & M & $\begin{array}{l}\text { Small intestine } \\
\text { diverticulum }\end{array}$ & NA & NA & 1 & 1 & NA & No & Conservative \\
\hline Xu XQ [29] & 2014 & 86 & M & $\begin{array}{l}\text { Small intestine } \\
\text { diverticulitis }\end{array}$ & 50 & NA & Multiple & NA & NA & Yes & $\begin{array}{l}\text { Open surgery with } \\
\text { resection }\end{array}$ \\
\hline Fresow R [30] & 2014 & 73 & M & $\begin{array}{l}\text { Small intestine } \\
\text { diverticulitis }\end{array}$ & 0 & NA & 3 & 0 & NA & Yes & $\begin{array}{l}\text { Open surgery, no } \\
\text { resection }\end{array}$ \\
\hline Corcelles R [31] & 2014 & 63 & $\mathrm{~F}$ & $\begin{array}{l}\text { Intestinal } \\
\text { perforation }\end{array}$ & NA & NA & Multiple & NA & NA & Yes & $\begin{array}{l}\text { Laparoscopic } \\
\text { resection }\end{array}$ \\
\hline Ojili V [32] & 2014 & 75 & M & $\begin{array}{l}\text { Small intestine } \\
\text { diverticulitis }\end{array}$ & NA & NA & NA & NA & NA & Yes & $\begin{array}{l}\text { Open surgery with } \\
\text { resection }\end{array}$ \\
\hline Zamani A [33] & 2013 & 63 & $\mathrm{~F}$ & $\begin{array}{l}\text { Small intestine } \\
\text { diverticulitis }\end{array}$ & 12 & NA & Multiple & 1 & NA & Yes & $\begin{array}{l}\text { Open surgery with } \\
\text { resection }\end{array}$ \\
\hline Aydin I [34] & 2013 & 74 & $\mathrm{~F}$ & $\begin{array}{l}\text { Small intestine } \\
\text { diverticulitis }\end{array}$ & $40-100$ & Mesentery & Multiple & 1 & False & Yes & $\begin{array}{l}\text { Open surgery with } \\
\text { resection }\end{array}$ \\
\hline Singal R [35] & 2012 & 63 & M & Unknown & NA & NA & Multiple & 0 & NA & No & $\begin{array}{l}\text { Open surgery, no } \\
\text { resection }\end{array}$ \\
\hline $\begin{array}{l}\text { Ferreira-Aparicio } \\
\text { FE [36] }\end{array}$ & 2012 & 65 & $\mathrm{~F}$ & Appendicitis & 0 & NA & Multiple & Multiple & NA & Yes & $\begin{array}{l}\text { Open surgery with } \\
\text { resection and } \\
\text { ileostomy }\end{array}$ \\
\hline Ferrarese A [37] & 2012 & 92 & $\mathrm{~F}$ & $\begin{array}{l}\text { Intestinal } \\
\text { perforation }\end{array}$ & NA & NA & NA & 1 & NA & Yes & $\begin{array}{l}\text { Open surgery with } \\
\text { resection }\end{array}$ \\
\hline Garnet DJ [38] & 2011 & 80 & M & $\begin{array}{l}\text { Small intestine } \\
\text { diverticulitis }\end{array}$ & NA & NA & Multiple & 1 & NA & Yes & $\begin{array}{l}\text { Laparoscopic } \\
\text { converted open } \\
\text { surgery, with } \\
\text { resection }\end{array}$ \\
\hline Tan KK [39] & 2011 & 88 & M & $\begin{array}{l}\text { Gastrointestinal } \\
\text { hemorrhage }\end{array}$ & NA & NA & NA & 0 & NA & No & $\begin{array}{l}\text { Open surgery with } \\
\text { resection }\end{array}$ \\
\hline Tan KK [39] & 2011 & 72 & M & $\begin{array}{l}\text { Gastrointestinal } \\
\text { hemorrhage }\end{array}$ & NA & NA & NA & 0 & NA & No & $\begin{array}{l}\text { Open surgery with } \\
\text { resection }\end{array}$ \\
\hline Tan KK [39] & 2011 & 84 & M & $\begin{array}{l}\text { Gastrointestinal } \\
\text { hemorrhage }\end{array}$ & NA & NA & Multiple & 0 & NA & No & $\begin{array}{l}\text { Open surgery with } \\
\text { resection }\end{array}$ \\
\hline Tan KK [39] & 2011 & 70 & M & $\begin{array}{l}\text { Intestinal } \\
\text { inflammation }\end{array}$ & NA & NA & Multiple & 1 & NA & Yes & $\begin{array}{l}\text { Open surgery with } \\
\text { resection }\end{array}$ \\
\hline Tan KK [39] & 2011 & 84 & M & $\begin{array}{l}\text { Intestinal } \\
\text { inflammation }\end{array}$ & NA & NA & 1 & 1 & NA & Yes & $\begin{array}{l}\text { Open surgery with } \\
\text { resection }\end{array}$ \\
\hline Tan KK [39] & 2011 & 75 & M & $\begin{array}{l}\text { Intestinal } \\
\text { inflammation }\end{array}$ & NA & NA & Multiple & 1 & NA & Yes & $\begin{array}{l}\text { Open surgery with } \\
\text { resection }\end{array}$ \\
\hline Nonose R [40] & 2011 & 86 & F & $\begin{array}{l}\text { Intestinal } \\
\text { perforation }\end{array}$ & $15-50$ & $\begin{array}{l}\text { Anti- } \\
\text { mesenteric }\end{array}$ & Multiple & 1 & NA & Yes & $\begin{array}{l}\text { Open surgery with } \\
\text { resection }\end{array}$ \\
\hline Falidas E [41] & 2011 & 55 & M & $\begin{array}{l}\text { Small intestine } \\
\text { diverticulum and } \\
\text { bowel obstruction }\end{array}$ & NA & NA & Multiple & 0 & NA & Yes & $\begin{array}{l}\text { Failed conservative, } \\
\text { open surgery with } \\
\text { resection }\end{array}$ \\
\hline Sakpal SV [42] & 2010 & 25 & $\mathrm{~F}$ & Enteritis & NA & Mesentery & 1 & 1 & NA & Yes & $\begin{array}{l}\text { Open surgery with } \\
\text { resection }\end{array}$ \\
\hline França M [43] & 2010 & 75 & M & $\begin{array}{l}\text { Small intestine } \\
\text { diverticulitis }\end{array}$ & NA & Mesentery & 3 & 1 & NA & Yes & $\begin{array}{l}\text { Open surgery with } \\
\text { resection }\end{array}$ \\
\hline Vanrykel F [44] & 2010 & 79 & F & $\begin{array}{l}\text { Small intestine } \\
\text { diverticulitis }\end{array}$ & NA & NA & Multiple & 1 & NA & Yes & $\begin{array}{l}\text { Laparoscopic } \\
\text { converted open } \\
\text { surgery, with } \\
\text { resection }\end{array}$ \\
\hline Chugay P [45] & 2010 & 89 & $\mathrm{~F}$ & $\begin{array}{l}\text { Small intestine } \\
\text { diverticula }\end{array}$ & NA & NA & Multiple & NA & NA & No & $\begin{array}{l}\text { Failed conservative, } \\
\text { open surgery with } \\
\text { resection }\end{array}$ \\
\hline Chugay P [45] & 2010 & 79 & M & $\begin{array}{l}\text { Small intestine } \\
\text { perforation }\end{array}$ & NA & NA & Multiple & 1 & NA & Yes & $\begin{array}{l}\text { Open surgery with } \\
\text { resection }\end{array}$ \\
\hline
\end{tabular}

PubMed database was queried for studies published from January 1, 2010 to April 31, 2020, with English language restriction. Search strategy included the term "jejunal diverticulitis." Case series lacking patient-specific data were excluded

Lig ligament, No. number, $N A$ not available, $F$ female, $M$ male 


\section{Abbreviations}

CT: Computed tomography; BUN: Blood urea nitrogen; IV: Intravenous

\section{Acknowledgements}

None

\section{Authors' contributions}

Natasha Leigh: writing of manuscript Brianne Sullivan: writing of manuscript Roi Anteby: edits for manuscript revisions Susan Talbert: editing of manuscript The authors read and approved the final manuscript.

\section{Funding}

This research did not receive any specific grant from funding agencies in the public, commercial, or not-for-profit sectors.

\section{Availability of data and materials}

This is a case report, so there is no dataset. The data for the patient, however, is available upon request.

\section{Ethics approval and consent to participate}

An ethics approval committee was not required as this is a case report.

\section{Consent for publication}

Informed consent for publication of their clinical details and/or clinical images was obtained from the patient. A copy of the consent form is available for review by the Editor of this journal.

\section{Competing interests}

The authors declare that there are no competing interests in preparing this article.

\section{Author details}

'Department of General Surgery, Icahn School of Medicine at Mount Sinai St. Luke's Roosevelt Hospital, 425 West 59th Street, Suite 7B, New York, NY 10019, USA. ²Faculty of Medicine, Tel Aviv University, Tel Aviv, Israel.

\section{Received: 13 March 2020 Accepted: 29 June 2020}

\section{Published online: 06 July 2020}

\section{References}

1. Lebert P, Millet I, Ernst O, Boulay-Coletta I, Corno L, Taourel P, et al. Acute jejunoileal diverticulitis: multicenter descriptive study of 33 patients. AJR Am J Roentgenol. 2018;210(6):1245-51.

2. Patel VA, Jefferis $H$, Spiegelberg B, Iabal Q, Prabhudesai A, Harris S. Jejunal diverticulosis is not always a silent spectator: a report of 4 cases and review of the literature. World J Gastroenterol. 2008;14(38):5916-9.

3. Horesh N, Klang E, Gravetz A, Nevo Y, Amiel I, Amitai MM, et al. Jejunal diverticulitis. J Laparoendosc Adv Surg Tech A. 2016;26(8):596-9.

4. Sakpal SV, Fried K, Chamberlain RS. Jejunal Diverticulitis: A rare case of severe peritonitis. Case Rep Gastroenterol. 2010:4(3):492-7.

5. Kagolanu DC, Subhani M, Novick D, Rizvon K. Jejunal diverticulitis in a healthy 91-year-old man. BMJ Case Rep. 2018;2018.

6. Kongara KR, Soffer EE. Intestinal motility in small bowel diverticulosis: a case report and review of the literature. J Clin Gastroenterol. 2000;30(1):84-6.

7. Fidan N, Mermi EU, Acay MB, Murat M, Zobaci E. Jejunal diverticulosis presented with acute abdomen and diverticulitis complication: a case report. Pol J Radiol. 2015:80:532-5.

8. Prough $\mathrm{H}$, Jaffe $\mathrm{S}$, Jones B. Jejunal diverticulitis. Journal of Surgical Case Reports. 2019;2019(1):rjz005.

9. Gurala D, Idiculla PS, Patibandla P, Philipose J, Krzyzak M, Mukherjee I. Perforated jejunal diverticulitis. Case Reports in Gastroenterology. 2019;13(3): 521-5.

10. Mazahreh TS, Aleshawi AJ, Alorjani MS, Elayyan R, Al-Zoubi NA. Arteriovenous malformations within jejunal diverticulosis: case report and literature review. BMC Surgery. 2019;19(1):70

11. Fleres F, Viscosi F, Bertilone E, Mazzeo C, Cucinotta E. Therapeutic strategies for jejunal diverticulitis: our experience and a review of the recent literature. J Vis Surg. 2018:4:152.

12. Abdelaki A, Bhatt N, Gupta N, Li S, Abdelbaki S, Kumar Y, et al. Complicated jejunal diverticulosis: report of three cases and review of the literature. Connecticut Medicine. 2018;82(4).
13. Syllaios A, Koutras A, Zotos PA, Triantafyllou E, Bourganos N, Koura S, et al Jejunal diverticulitis mimicking small bowel perforation: case report and review of the literature. Chirurgia (Bucur). 2018;113(July $\neg$ August (4)):576-581.

14. Ejaz S, Vikram R, Stroehlein JR. Non-Meckel small intestine diverticulitis. Case Reports in Gastroenterology. 2017;11(2):462-72.

15. Grubbs J, Huerta S. Perforated jejunal diverticulitis in a nonagenarian veteran: a case report. International Journal of Surgery Case Reports. 2017; 40:77-9.

16. Kumar D. Complicated jejunal diverticulitis with unusual presentation. Radiology Case Reports. 2018;13(1):58-64.

17. Cui J, Mehanna D. Jejunal diverticulitis secondary to enterolith: a case report. ANZ Journal of Surgery. 2019;89(6):E261-E2.

18. Malghan L, Uttley A, Scullion D. Small bowel obstruction due to a jejunal enterolith: an unusual complication of jejunal diverticulitis. Case Reports. 2017;2017.

19. Karas L, Asif M, Chun V, Khan FA. Complicated small bowel diverticular disease: a case series. Case Reports. 2017;2017:bcr-2017-219699.

20. Mohi RS, Moudgil A, Bhatia SK, Seth K, Kaur T. Complicated jejunal diverticulosis: small bowel volvulus with obstruction. Iranian Journal of Medical Sciences. 2016;41(6):548.

21. Aydın E, Yerli H, Avcl T, Yılmaz T, Gülay H. One of the rare causes of acute abdomen leading to subileus: jejunal diverticulitis. Balkan Medical Journal. 2016:33(3):354.

22. Tenreiro N, Moreira H, Silva S, Marques R, Monteiro A, Gaspar J, et al. Jejunoileal diverticulosis, a rare cause of ileal perforation-case report. Annals of Medicine and Surgery. 2016;6:56-9.

23. Ghrissi $R$, Harbi $H$, Elghali MA, Belhajkhlifa MH, Letaief MR. Jejunal diverticulosis: a rare case of intestinal obstruction. Journal of Surgical Case Reports. 2016;2016(2).

24. Harbi H, Fendri S, Sahnoun M, Amar MB, Mzali R. Fatal acute peritonitis due to perforated jejunal diverticulum. Presse medicale (Paris, France: 1983). 2017:46(2 Pt 1):246.

25. De Minicis S, Antonini F, Belfiori V, Cascio ML, Marraccini B, Piergallini S, et al. Small bowel diverticulitis with severe anemia and abdominal pain World Journal of Clinical Cases: WJCC. 2015;3(5):462.

26. Natarajan K, Phansalkar M, RGb V Thangiah G. Jejunal diverticulosis with perforation-a challenging differential diagnosis of acute abdomen: case report. Journal of Clinical and Diagnostic Research: JCDR. 2015:9(2):ED03.

27. Kassir R, Boueil-Bourlier A, Baccot S, Abboud K, Dubois J, Petcu CA, et al. Jejuno-ileal diverticulitis: etiopathogenicity, diagnosis and management. International Journal of Surgery Case Reports. 2015;10:151-3.

28. Levack MM, Madariaga ML, Kaafarani HM. Nonoperative successful management of a perforated small bowel diverticulum. World Gastroenterol. 2014;20:18477-9.

29. $X u$ XQ, Hong T, Li BL, et al. Active gastrointestinal diverticulum bleeding diagnosed by computed tomography angiography. World J Gastroenterol. 2014:20:13620-4.

30. Fresow $\mathrm{R}$, Vieweg $\mathrm{H}$, Kamusella $\mathrm{P}$, et al. Jejunal diverticulitis ascending to the duodenum as a rare cause of acute abdomen. J Clin Diagn Res. 2014;8: RD07-8.

31. Corcelles R, Pavel M, Lacy A. Perforated small bowel diverticulitis after gastric bypass. JSLS: Journal of the Society of Laparoendoscopic Surgeons. 2014 Jan;18(1):142.

32. Ojili V, Parizi M, Gunabushanam G. Timely diagnosis of perforated jejuna diverticulitis by computed tomography. J Emerg Med 2013:44:614-6. 46 Corcelles R, Pavel M, Lacy A. Perforated small bowel diverticulitis after gastric bypass. JSLS 2014;18:142-145.

33. Zamani A, Peters CJ, Chadwick SJ. The use of an ex vivo contrast study at the time of surgery to confirm the site of a perforated jejunal diverticulum. BMJ Case Rep. 2013;2013. https://doi.org/10.1136/bcr-2012-006788.

34. Aydin I, Pergel A, Yucel AF, et al. A rare cause of acute abdomen: jejuna diverticulosis with perforation. J Clin Imaging Sci. 2013:3:31.

35. Singal R, Gupta S, Airon A. Giant and multiple jejunal diverticula presenting as peritonitis a significant challenging disorder. J Med Life. 2012;5:308-10.

36. Ferreira-Aparicio FE, Gutiérrez-Vega R, Gálvez-Molina Y, et al. Diverticular disease of the small bowel. Case Rep Gastroenterol. 2012;6:668-76.

37. Ferrarese A, Assoua C, Ahmad K, et al. Perforated jejunal diverticulitis. Clin Res Hepatol Gastroenterol. 2012;36:99-100.

38. Garnet DJ, Scalcione LR, Barkan A, et al. Enterolith ileus: liberated large jejunal diverticulum enterolith causing small bowel obstruction in the setting of jejunal diverticulitis. Br J Radiol. 2011;84:e154-7. 
39. Tan KK, Liu JZ, Ho CK. Emergency surgery for jejunal diverticulosis: our experience and review of literature. ANZ I Surg. 2011;81:358-61.

40. Nonose R, Valenciano JS, de Souza Lima JS, et al. Jejunal diverticular perforation due to enterolith. Case Rep Gastroenterol. 2011:5:445-51.

41. Falidas E, Vlachos K, Mathioulakis S, et al. Multiple giant diverticula of the jejunum causing intestinal obstruction: report of a case and review of the literature. World J Emerg Surg. 2011;6:8.

42. Sakpal SV, Fried K, Chamberlain RS. Jejunal diverticulitis: a rare case of severe peritonitis. Case Rep Gastroenterol. 2010;4:492-7.

43. França M, Certo M, Silva D, et al. Elderly patient with acute, left lower abdominal pain: perforated jejunal diverticulitis (2010:7b). Eur Radiol. 2010; 20:2541-5.

44. Vanrykel F, Pattyn P, Vuylsteke P, et al. Perforated jejunal diverticulitis: a rare presentation of acute abdomen. Acta Chir Belg. 2010;110:367-9.

45. Chugay P, Choi J, Dong XD. Jejunal diverticular disease complicated by enteroliths: report of two different presentations. World J Gastrointest Surg. 2010;2:26-9.

46. Nejmeddine A, Bassem A, Mohamed H, Hazem BA, Ramez B, Issam BM. Complicated jejunal diverticulosis: a case report with literature review. N Am J Med Sci. 2009;1 (4):196-9.

47. Sioulas AD, Polymeros D, Beis D, Triantafyllou K. Jejunal diverticulosis. Ann Gastroenterol. 2013;26(4):354.

48. Chendrasekhar A, Timberlake GA. Perforated jejunal diverticula: an analysis of reported cases. Am Surg. 1995;61(11):984-8.

\section{Publisher's Note}

Springer Nature remains neutral with regard to jurisdictional claims in published maps and institutional affiliations.

\section{Submit your manuscript to a SpringerOpen ${ }^{\circ}$ journal and benefit from:}

- Convenient online submission

- Rigorous peer review

- Open access: articles freely available online

- High visibility within the field

- Retaining the copyright to your article 